\title{
Respuesta a los comentarios sobre Categorías y autoconciencia en Kant
}

\author{
PEDRO STEPANENKO \\ Instituto de Investigaciones Filosóficas \\ Universidad Nacional Autónoma de México \\ pedros@servidor.unam.mx
}

\begin{abstract}
RESUMEN: El autor de Categorías y autoconciencia en Kant responde a los comentarios de I. Cabrera, E. Lazos y G. Leyva. En respuesta al comentario de este último, caracteriza su interpretación de la deducción kantiana de las categorías como reconstrucción de una discusión entre distintas posiciones y expresa su desconfianza ante una reconstrucción sistemática de todas sus partes. En su respuesta al comentario de E. Lazos, analiza los distintos conceptos de objetividad que Kant maneja en la deducción trascendental y que desembocan en el problema de las representaciones subjetivas. En su respuesta al comentario de I. Cabrera aclara en qué sentido considera que la llamada deducción subjetiva puede verse como un argumento analítico-regresivo y por qué el concepto de autoconciencia como autoadscripción de representaciones no puede servir para argumentar a favor de la necesidad de las categorías.
\end{abstract}

PALABRAS ClAVE: Kant, deducción trascendental, interpretación, objetividad, autoconciencia

Se suele comparar el vacío que provoca terminar un libro con la depresión posparto. Ojalá todos los que terminamos un libro pudiéramos hacer esa comparación: estaríamos seguros de que aquello que tanto tiempo nos ha ocupado seguirá teniendo vida. Pero, el vacío que provoca terminar un libro viene acompañado o se confunde precisamente con el temor de que aquello que nos ha ocupado tanto tiempo desaparezca en el olvido o en lo que Carlos Pereda llama el ninguneo. En este contexto, es un privilegio tener lectores como los que han comentado previamente mi libro. Los tres conocen muy bien los problemas en los que me he metido y la diversidad de sus intereses los ha hecho enfocar distintas partes de mi texto. El comentario de Gustavo Leyva se concentra en la reconstrucción de la génesis de la deducción trascendental que presento en los tres primeros capítulos y que está basada en las investigaciones que Wolfgang Carl expone en Der schweigende Kant. El comentario de Efraín Lazos ataca, quizá porque me conoce bien, el asunto que más me preocupa de mi libro: lo que Bernhard Thöle llama "el problema de las representaciones subjetivas", ${ }^{1}$ es decir, el rechazo de las representaciones que no pueden articularse objetivamente

1 Cfr. B. Thöle, Kant und das Problem der Gesetzmäßigkeit der Natur, W. de Gruyter, Berlín, 1991, pp. 64-69. 
con otras representaciones. De acuerdo con el quinto capítulo, el más largo y propositivo, Kant se ve obligado, en la primera edición de la Crítica de la razón pura, a rechazar este tipo de representaciones por querer fusionar los dos proyectos de deducción que expongo en los tres primeros capítulos. Isabel Cabrera, aun cuando ofrece un panorama general del libro, concentra sus inquietudes en la posición que adopto, en los dos apéndices, frente a algunas interpretaciones contemporáneas de la deducción trascendental. Como el orden en que he mencionado los comentarios corresponde a la disposición de los temas en el libro, responderé en ese orden.

La preocupación predominante del comentario de Gustavo Leyva es la de ubicar la perspectiva desde la cual interpreto los textos de Kant. Ya que uno de mis objetivos es exponer los distintos proyectos de deducción previos a las versiones publicadas en la Crítica de la razón pura y, para ello, me baso en las investigaciones realizadas por W. Carl en Der schweigende Kant, Leyva se detiene a presentar la propuesta interpretativa de este último. Wolfgang Carl, como lo señala Leyva, se aparta, por un lado, de una interpretación selectiva como la de P.F. Strawson y, por el otro, de una interpretación que busque acabar con las oscuridades del texto ofreciendo una reconstrucción consistente con todas las partes del mismo, como la que intenta llevar a cabo Dieter Henrich. A diferencia de ellos, lo que pretende hacer W. Carl en su libro es comprender la deducción mediante la historia de su desarrollo, mediante un estudio de las preocupaciones que llevaron al autor a ese texto, con esas oscuridades y esa complejidad que, de hecho, tiene. No sin cierta razón, G. Leyva se pregunta si esto no equivale a insistir en el ingenuo lema del retorno al texto. En defensa de Carl, hay que decir que él mismo toma distancia de ese lema y que, en contraste con éste, señala que lo que busca es sólo hacer comprensible el texto de Kant como "expresión de las intenciones argumentativas de su autor", las cuales se pueden encontrar en las notas previas a la Crítica de la razón pura. ${ }^{2}$ Es cierto que, al distinguir sus objetivos de los que sustenta la interpretación de D. Henrich, afirma que pretende guiarse por la penetración (Einsicht) del autor interpretado y no por la de aquel que interpreta. ${ }^{3}$ Esto puede resultar ingenuo. Sin embargo, yo creo que hay que ver en el libro de W. Carl no una interpretación acabada, sino un momento en el proceso de interpretación: aquel en el cual el texto se resiste a satisfacer las expectativas del que interpreta. Como G. Leyva lo menciona en su comentario, la expectativa original de W. Carl era leer la deducción trascendental de las categorías como una discusión con

2 Cfr. W. Carl, Der schweigende Kant. Die Entwürfe zu einer Deduktion der Kategorien vor 1781, Vandenhoeck \& Ruprecht, Gotinga, 1989, p. 14.

3 Ibid. 
el naturalismo de Hume. Si esa expectativa era acertada, una investigación de la génesis de este texto en los doce años previos a la publicación de la Crítica enriquecería la comprensión de la posición trascendental como alternativa del naturalismo. Sin embargo, los textos que dan testimonio del desarrollo filosófico de Kant en esos doce años no adquieren sentido bajo esa hipótesis. Era, pues, necesario rectificar esa expectativa. El libro de W. Carl puede verse, entonces, como un análisis que abre la posibilidad de conformar una nueva unidad de sentido, una unidad que permita articular las distintas líneas argumentativas de la deducción trascendental. Esta articulación quizá no sea la de una teoría acabada de la intencionalidad. Quizá el sentido de la deducción es el de un proyecto o el de una discusión entre distintas posiciones.

El libro de W. Carl distingue tres proyectos de deducción trascendental y permite comprender las preocupaciones que llevaron a Kant a buscar su solución en cada uno de ellos. De esta manera, W. Carl no cae en los análisis, frecuentes en cierta tradición filosófica, que terminan por desbaratar el texto y reducirlo a una serie de pensamientos inconexos, cuya unidad no puede atribuirse más que a las confusiones del autor. Pero tampoco se empeña en dar con una versión redonda del texto, en la cual todas sus piezas embonen a la perfección. Encuentra, pues, un sano término medio y parece suscribir, así, la opinión de Kemp Smith acerca del mayor mérito filosófico de Kant, a saber: su capacidad para reconocer las dificultades de cada una de las soluciones que era capaz de proponer para resolver los problemas que se había planteado.

El método de trabajo de Kant parece haber consistido en alternar con varias soluciones posibles, desarrollando cada una en su turno, con la esperanza de encontrar una posición intermedia que comparta los méritos de todas. Cuando esa posición intermedia no surge, como sucede con frecuencia, Kant desarrolla su pensamiento a través de las líneas paralelas de las perspectivas alternas. ${ }^{4}$

Lo que me atrajo del libro de W. Carl fue precisamente ese término medio entre un análisis sin dirección y una ingenua reconstrucción sistemática de todas las piezas de la deducción. Ahora, lo que yo intenté hacer en mi libro fue desarrollar el potencial interpretativo de la distinción de proyectos que Carl establece en el suyo. Hago uso de esa distinción para explicar los conflictos y las tensiones entre las distintas líneas argumentativas de la deducción en la primera edición de la Crítica de la razón pura. En lugar de distinguir tres proyectos, yo destaco básicamente dos para enfatizar la

4 N. Kemp Smith, A Commentary to Kant's "Critique of pure Reason”, 2a. ed., Macmillan, Nueva York, 1923, p. xxii. 
tensión entre ellos. En la exposición del segundo, me aparto de Carl para destacar una de las ideas centrales de una de las líneas argumentativas de la deducción en la Crítica, a saber: que la unidad que constituyen las categorías es la unidad de la autoconciencia. El problema que ubico como foco de esas tensiones es lo que Bernhard Thöle llama "el problema de las representaciones subjetivas", es decir, el rechazo de todas aquellas representaciones que no estén articuladas objetivamente conforme a las categorías. Este problema puede considerarse como el foco de esas tensiones porque, de acuerdo con el primer proyecto de deducción, la diferencia entre representaciones objetivas y representaciones subjetivas es un presupuesto que nos permite especificar el conjunto de conceptos o reglas que estructuran objetivamente nuestras representaciones; mientras que, de acuerdo con el otro proyecto, la conciencia misma de cada representación implica su articulación objetiva. Las tensiones, por supuesto, se generan por querer fusionar los dos proyectos, en particular, por querer identificar las categorías como conceptos que determinan lo que es un objeto en general con las categorías como reglas que permiten integrar todas nuestras representaciones en una única conciencia. Son muchos los problemas que están involucrados en estas tensiones. Algunos de ellos son cuestiones que ocupan la atención de muchos filósofos de la mente y epistemólogos contemporáneos. Aquí sólo podré mencionar algunas: si la diferencia entre cómo pienso que son las cosas y cómo me parece que son es esencial para una concepción satisfactoria de la conciencia y del conocimiento, si hay contenidos no-conceptuales de la conciencia, si todos los actos de conciencia presuponen cierta normatividad. Me atrevo, pues, a decir que un análisis de las tensiones y conflictos que se encuentran en la deducción trascendental contribuye a una mejor comprensión de estos problemas contemporáneos. Es cierto que no es a partir de la formulación contemporánea de estos problemas como me acerco a la deducción, sino que los abordo siempre desde una perspectiva interna a la filosofía de Kant. Por esto, es legítima la preocupación de G. Leyva cuando se pregunta si mi manera de leer a Kant no resulta demasiado inmanente y ligada al texto. En mi libro, efectivamente, no pretendo ir más allá de la filosofía de Kant. Sin embargo, no hay que olvidar que los problemas filosóficos contemporáneos sólo tienen sentido por su articulación con la historia de la filosofía. Querer atacar esos problemas sin tener en cuenta el trasfondo histórico que hace posible incluso su formulación es tan ingenuo como querer comprender un texto filosófico de la tradición haciendo abstracción de nuestro propio punto de vista. No hay que olvidar la advertencia con la cual W. Sellars empieza su libro Science and Metaphysics y con la cual estoy seguro que G. Leyva 
coincide: "La filosofía sin la historia de la filosofía si no es vacía o ciega, al menos es tonta." 5

Paso ahora a responder los comentarios de Efraín Lazos, quien se pregunta si el problema que yo destaco como el punto en el cual chocan los dos proyectos de deducción es, en realidad, un problema. Si no estoy confundido, lo que se pregunta E. Lazos es si el rechazo de las representaciones subjetivas, el rechazo de las representaciones que no están relacionadas mediante categorías con otras representaciones, constituye un problema para lo que podríamos llamar la teoría de la intencionalidad de Kant. Si lo que Kant hace en la Doctrina trascendental de los elementos es sólo caracterizar lo que es el contenido de un pensamiento, podemos perfectamente hacer abstracción de aquellos aspectos de los estados mentales que no forman parte del contenido. Visto así, el problema de las representaciones subjetivas parece, efectivamente, irrelevante. Pero E. Lazos da por sentado precisamente lo que en mi libro está en cuestión, aunque lo formule en otros términos, a saber: si Kant maneja el concepto de objetividad como mera intencionalidad o si caracteriza la objetividad de tal manera que presupone el concepto de verdad.

Cuando C.I. Lewis se pregunta, en Mind and the World-Order, si el sabio de Königsberg no tenía sueños, concibe los principios que se derivan de las categorías como principios que delimitan lo que podemos considerar como real y señala que Kant confundió estos principios con los principios que determinan la posibilidad de la experiencia. C.I. Lewis insiste en que no podemos limitar a priori la experiencia; lo que podemos determinar a priori es lo que puede ser un objeto real. Sabemos a priori el tipo de relaciones que deben tener los contenidos de nuestras experiencias para considerarlos como objetos reales. Pero no podemos determinar a priori si todos los contenidos de nuestras experiencias concuerdan con ese tipo de relaciones. El contenido de los sueños o de las alucinaciones, de acuerdo con C.I. Lewis, no responde a las relaciones categoriales. ${ }^{6}$ Ahora bien, si las relaciones categoriales definen la objetividad de nuestras experiencias, entonces el contenido de esos estados mentales es subjetivo y, por lo tanto, los requisitos de contenido no pueden coincidir con los requisitos de objetividad.

Lo que Bernhard Thöle llama el problema de las representaciones subjetivas tiene su origen en esta interpretación de las relaciones categoriales. Yo creo que esta interpretación es correcta si se toma en consideración el primer

5 W. Sellars, Science and Metaphysics, Routledge and Kegan Paul, Londres 1968, p. 1.

${ }^{6}$ Cfr. C.I. Lewis, Mind and the World-Order, Charles Sribner's Sons, Nueva York, 1929, pp. 221-222. 
proyecto de deducción que expongo en mi libro. Pero entra en tensión con el segundo proyecto, para el cual las relaciones categoriales resultan ser incluso condiciones para tener conciencia de las intuiciones relacionadas. Querer fusionar ambos proyectos es lo que lleva al absurdo de negar la existencia de los contenidos de estados mentales como los que tenemos en los sueños o en las alucinaciones. ¿Quiere esto decir que los principios que se derivan de las categorías establecen, de acuerdo con el primer proyecto, condiciones de verdad, mientras que en el segundo tan sólo establecen condiciones de objetividad, entendida esta última como mera intencionalidad? Sí, quizá ésta sea una buena manera de plantear el problema, siempre y cuando las condiciones de verdad en el primer proyecto se entiendan sólo como condiciones negativas de la verdad. Lo que quizá resulte desconcertante es que a estas condiciones negativas de la verdad podemos también llamarlas condiciones de objetividad. En efecto, de acuerdo con el primer proyecto, la objetividad puede ser considerada, en un primer momento, propiedad de los juicios o de los pensamientos, entendiendo estos últimos como la contraparte de las intuiciones. La objetividad tiene que ver, en este caso, con la forma en que están relacionados los términos o los conceptos en los juicios y lo que garantiza es que a través de esos juicios pensemos en objetos reales. Ciertas intuiciones o experiencias, claro está, son las que determinan el valor de verdad del juicio; pero, para que esto suceda, el juicio tiene que estar estructurado conforme a categorías. Esta propiedad originaria de los juicios, puede, sin embargo, aplicarse a los contenidos de los estados mentales. En este caso, significa que esos contenidos están relacionados de tal manera que pueden determinar el valor de verdad de un juicio objetivo. Lo pueden hacer de manera positiva o negativa, es decir, las relaciones entre los elementos del contenido pueden hacer que un juicio objetivo sea verdadero o falso. Está claro, pues, que, siguiendo el primer proyecto de deducción, la objetividad no coincide ni con la mera intencionalidad, ya que puede haber contenidos que no determinen el valor de verdad de un juicio objetivo, ni con la verdad, puesto que los juicios objetivos pueden ser verdaderos o falsos.

Pero Kant no sólo maneja este sentido fuerte de objetividad, también maneja el concepto de objetividad como mera intencionalidad. Este sentido amplio de objetividad es el que interviene en el segundo proyecto de deducción. Las categorías ya no sólo son aquí los conceptos a través de los cuales pensamos en un objeto real, sino también funciones que sintetizan nuestras intuiciones de tal manera que los contenidos de nuestros estados mentales formen parte de la unidad de la conciencia. El problema de las representaciones subjetivas surge, pues, por querer identificar dos sentidos del término objetividad o dos maneras distintas de caracterizar las categorías. 
Yo creo que Kant, en la Crítica de la razón pura, lleva a cabo explícitamente esta identificación cuando afirma lo siguiente:

La misma función que da unidad a las distintas representaciones en un juicio proporciona también a la mera síntesis de diferentes representaciones en una intuición una unidad que, en términos generales, se llama concepto puro del entendimiento. (A79-80/ B104-105) ${ }^{7}$

Kant nunca renuncia al sentido fuerte de objetividad, de tal manera que el objetivo de la deducción subjetiva, la parte de la deducción que pretende mostrar la necesidad de las categorías, puede formularse en los siguientes términos: mostrar que el contenido de nuestras experiencias no puede ser más que un objeto real, ya que las mismas funciones de síntesis que hacen de las intuiciones objetos de la conciencia son las que, en términos de C.I. Lewis, nos permiten pensar en un objeto real. Esta posición puede considerarse escandalosa o inofensiva; lo cierto es que elimina la diferencia entre cómo me parece que son las cosas y cómo pienso que son. Puede resultar inofensiva si se reformula en los siguientes términos: el contenido de nuestras experiencias tiene que estar estructurado de tal manera que pueda determinar el valor de verdad de algún juicio objetivo. El contenido de nuestras experiencias es algo real; el problema es dar con los juicios objetivos que puedan contrastarse con ese contenido. Lo que yo me preguntaría aquí es cómo debemos entender el contenido de nuestras experiencias mientras no contemos con los juicios objetivos correspondientes. Éste es, en realidad, el aspecto del problema de las representaciones subjetivas que me preocupa en mi libro y, por ello, me parece atractiva la reformulación de la postura de Kant en la segunda edición de la Crítica de la razón pura. Esta postura puede interpretarse de tal manera que se exija de todo contenido no que sea objetivo por el hecho de que tengamos conciencia del mismo, sino que lo sea porque, en principio, debemos ser capaces de encontrar los juicios objetivos que nos permitan ubicarlo en una visión del mundo real.

Este último punto está íntimamante ligado con la primera de las inquietudes que expone Isabel Cabrera en su comentario: si las representaciones subjetivas, aquellas que no pueden considerarse representaciones de objetos reales que existan con independencia del sujeto, no son también objetivas en tanto estados mentales particulares de las personas. De acuerdo con la reformulación de la postura de Kant, en la segunda edición de la Crítica, esta observación sería perfectamente factible. Si el carácter subjetivo de una

7 I. Kant, Crítica de la razón pura, trad. de Pedro Ribas, Alfaguara, Madrid, 1995, pp. 112-113. 
representación se debe a que no puede determinar el valor de verdad de juicios objetivos que se refieren a objetos reales que existen con independencia del sujeto, entonces debemos buscar juicios objetivos que se refieran a otro campo de la realidad. Nuestros propios estados mentales, por ejemplo. Nuestras representaciones pueden ocupar distintas posiciones en la imagen del mundo real que nos vamos formando. Sin embargo, la manera en que I. Cabrera expresa su inquietud puede conducir a un malentendido, a una confusión entre los estados mentales y sus contenidos. Una confusión que E. Lazos advierte cuando menciona los diferentes significados del término "representación" en Kant. Este término puede significar el contenido de un estado mental o el estado mental que tiene ese contenido. Si la observación de I. Cabrera lo que señala es que el contenido de un estado mental es objetivo porque se puede adjudicar a una persona en tanto estado mental, entonces está confundiendo los dos significados mencionados. El peligro de esta confusión es que bajo ella podemos transferir las propiedades del contenido al estado mental y decir, por ejemplo, que si sueño con un elefante rosa, entonces mi estado mental es de color rosa y tiene la forma de un elefante. Si la observación de I. Cabrera, en cambio, respeta la diferencia entre un estado mental y su contenido, entonces lo que señala es que el contenido de un estado mental muestra su objetividad (en sentido fuerte) cuando determinamos objetivamente el estado mental. Así interpretada, esta observación me parece que coincide con la posición de la segunda edición de la Crítica, con aquella posición que, de acuerdo con mi libro, logra evitar los conflictos más agudos entre la deducción objetiva y la deducción subjetiva. Sin embargo, no veo cómo la observación de I. Cabrera sea una objeción en contra de la deducción objetiva por sí misma, ya que si hacemos abstracción de la deducción subjetiva, podría decirse que la objetivación de un estado mental deja intacto el contenido del estado mental. Para argumentar en contra de esto, Kant requiere todo el andamiaje de la deducción subjetiva.

En cuanto a la segunda preocupación de I. Cabrera, si la deducción subjetiva puede considerarse un argumento analítico-regresivo, estoy básicamente de acuerdo con ella. Estoy de acuerdo en que la deducción subjetiva puede verse como un argumento que busca determinar las condiciones de posibilidad de la autoconciencia o de la experiencia, caracterizada simplemente como una pluralidad de representaciones. El problema que me preocupa en el primer apéndice de mi libro son las limitaciones que se le han atribuido a los resultados que se pueden obtener de estos argumentos. Muchos autores consideran que estos argumentos sólo pueden probar las condiciones de posibilidad de un tipo de conocimiento o de la experiencia entendida de una manera específica. No pueden, por ello, determinar las condiciones necesarias de la experiencia o del conocimiento en general, pues su punto 
de partida deja abierta la posibilidad de otro tipo de conocimiento o de una caracterización diferente de la experiencia. En la deducción objetiva, Kant parte de una descripción particular de la experiencia como conocimiento de objetos y, por esto, se puede decir que "parte de lo que investiga como si fuese dado". Sus resultados están, por lo tanto, limitados a esa descripción de la experiencia. Ésta es la razón por la cual no se le puede considerar un argumento antiescéptico. El escéptico puede aún ofrecer otra caracterización de la experiencia y, consecuentemente, negarse a aceptar las condiciones de posibilidad que se han obtenido como condiciones necesarias de la experiencia en general. La deducción subjetiva, en cambio, tiene que partir de una caracterización lo suficientemente amplia de la experiencia para que sus resultados puedan valer para cualquier descripción posible de la experiencia. En este caso, no puede decirse que "se parte de lo que se investiga como si fuese dado". Sus premisas no deben contener una descripción específica de la experiencia que requiera de una ulterior justificación. En este sentido, sus premisas pueden considerarse principios, que es precisamente lo que define al método sintético-progresivo. El escéptico puede exigir una justificación de la experiencia entendida como conocimiento de objetos; pero ya no podría hacerlo si consideramos a la experiencia simplemente como una pluralidad de representaciones subjetivas.

Resumiendo: si los argumentos analítico-regresivos se definen sólo como argumentos que buscan determinar las condiciones de posibilidad de algo que hemos aceptado, estoy de acuerdo en considerar a la deducción subjetiva como un argumento analítico-regresivo. Pero, si se le añade a esa definición que esos argumentos deben partir de lo que se investiga como si fuera dado, entonces no estoy de acuerdo.

Por último, I. Cabrera me advierte acerca del peligro que corro al interpretar la autoconciencia como conciencia de las reglas o de las funciones de síntesis que supuestamente garantizan la unidad e identidad de la conciencia, en lugar de entender la autoconciencia como autoadscripción de representaciones. El peligro es caer en una especie de "psicología trascendental", una psicología que habla de hechos psíquicos que ocurren en un misterioso espacio que no es el empírico.

Antes que nada, debo aclarar que acepto los dos sentidos del término "autoconciencia", como autoadscripción de representaciones y como conciencia de la unidad que conforman las reglas o funciones de síntesis. El problema es cuál de estos dos sentidos desempeña el papel central en la deducción subjetiva, en el argumento kantiano a favor de la necesidad de las categorías. El punto de partida de este argumento es que la conciencia de una pluralidad de representaciones presupone la conciencia de nuestra identidad, la conciencia de algo permanente a lo largo de la secuencia temporal en la que 
debe estar distribuida la pluralidad de representaciones. La autoadscripción de representaciones presupone nuestra propia identidad; pero en el mismo sentido en que hablamos de la identidad de un objeto. Presupone, desde la perspectiva de Kant, el uso de las categorías cuya necesidad se pretende probar. El escéptico, por lo tanto, puede rechazar la posibilidad de la autoadscrpción, la cual no es más que un caso particular de conocimiento de objetos, como la propia I. Cabrera señala en su primera observación. Hay que recurrir, pues, a otro sentido de identidad, no a la identidad de un objeto. La alternativa es precisamente la identidad del marco conceptual que nos permite darle unidad a nuestras representaciones. No puedo negar que haya oscuridades y dificultades en esta manera de entender la conciencia de nuestra identidad, pero me parece que son menores que las que pretende evitar.

La autoconciencia como conciencia de la unidad o del espacio en el cual relacionamos nuestras representaciones es una manera de entender lo que Kant denomina "autoconciencia trascendental". Otra manera de entenderlo es interpretar esa autoconciencia como conciencia del proceso de síntesis que condiciona nuestra receptividad. Yo creo que el peligro de caer en una psicología trascendental amenaza sobre todo a esta última manera de interpretar la autoconciencia trascendental, pues nos obliga a aceptar síntesis puras o trascendentales, hechos psíquicos trascendentales. En mi libro distingo estas dos nociones de autoconciencia trascendental y señalo que sólo la primera puede evitar el problema de las representaciones subjetivas. Pero también tiene la ventaja de evitar todas esas actividades trascendentales de las cuales habla Kant en la deducción. Bajo la primera interpretación, el término "trascendental" puede reservarse para la esfera conceptual, lo cual ya no resulta tan esotérico, y como adjetivo de la autoconciencia debe calificar los conceptos de los cuales se tiene conciencia, no designar alguna extraña propiedad de la conciencia.

Quedan, por supuesto, muchas cosas por aclarar. Hablar de conciencia nos lleva inevitablemente a plantear problemas de orden psicológico. Uno de esos problemas es encontrar una caracterización satisfactoria de la conciencia tácita. Sin ella, hablar de autoconciencia trascendental como condición de posibilidad de la conciencia empírica, de la conciencia común y corriente, seguirá pareciendo una extravagante pieza de museo. Determinar los distintos usos del término "conciencia" también es una tarea fundamental, previa al planteamiento de los problemas psicológicos. Mientras no se aclaren todas estas cuestiones, seguiremos lidiando con muchas oscuridades, pero también tenemos que avanzar a oscuras si queremos enfrentarlas. 